\title{
LESSONS LEARNED FROM THE 2022 WORLD CUP FINAL STADIUM, QATAR
}

\author{
AMBROSIO GÓMEZ MORALES \& CALLUM JOHN SYKES \\ AFL Architects, UK
}

\begin{abstract}
In this paper we detail the auditing process taken on the World Cup Final Stadium 2022 (80,000 seats), under extensive scrutiny for its high cost estimate. As a result of the efficiencies identified via this method, the client saved $40 \%$ on a $£ 2$ bn construction cost. Through detailed demonstrations of Dynamo scripts and BIM workflows, this article will pass on lessons learned from this intensive project. The ultimate objective will be to build on best practices used by all parties involved in the design process. The paper is relevant for any architect, programmer, project manager or those familiar with BIM software across the built environment. Adding to the combined BIM knowledge in the industry, the key takeaways discussed here will continue to save thousands of man-hours and millions of pounds for clients and contractors all over the world. The article will also demonstrate how efficient solutions can translate into multiple stadium configurations, including parameters such as seat numbers, bowl shape and pitch size. The authors currently manage AFL Architects' BIM department, and have been at the forefront of the practice's BIM innovations. Significant investment in research and development has directly led to procuring larger, more complex and higher-profile projects, demonstrating the fundamental importance of BIM in the UK construction industry.
\end{abstract}

Keywords: computational design, workflow, BIM, architecture, stadium, auditing, automation, methodology, management.

\section{INTRODUCTION}

The World Cup Final Stadium is an 80,000 capacity stadium in Doha, Qatar. AFL Architects were commissioned to interrogate the existing designs and make the more efficient, a task that required the involvement of countless staff, from six countries across the world.

The completion of the project resulted a comprehensive review and retool of our workflow processes and documentation toolchain. These changes not only resulted in a renewed ease of working, but also made training new starters much easier.

\section{OPERATIONAL PROCESS AND METHODOLOGY}

Architecture, by its very nature, is a highly creative and inventive industry. Yet with any creative endeavour, a project needs to be underpinned by controlled workflows and a clear process. This is especially important as client expectations rise and with it, a project's time and quality demands.

The operational process in architecture should work very much like a manufacturing plant, with each part of the production line having a specific task as part of a well configured workflow. As with every project in architecture, the timeline is very short and every action needs to be managed effectively. This is not to say Architecture should feel like a production line. It should not. It is a vastly creative and inventive industry, and it should feel like that.

If we were to watch a real production line, we would see a very clear flow of work, very clear positions for employees/machines and actions being repeated in an identical, reproducible manner. This is the end goal. Everything we do and everything we plan should be reproduceable and documented, otherwise the knowledge will be lost, and the task cannot be repeated. To conquer such inefficiencies and to allow us to scale our processes between 
our smallest and biggest jobs, we have thoroughly documented each process from the start to finish of a project at a coarse level of detail and work towards a more detailed workflow.

For now, let's assess the flow of processes and workflows in relation to the aforementioned manufacturing plant, with its many production lines.

\subsection{Business development manager}

In every company there will be a person who accepts or bids for jobs. This is of course a very necessary part and every company needs jobs to continue functioning. A key role of this person is to liaise with the various managers of the production line to ensure that they do not overload the production line. This is the crux of the entire operation: too little work and the company will not be able to exist, too much work and the company will not be able to function effectively, rendering all the workflows and processes obsolete as people are forced to cut corners to meet deadlines.

\subsection{BIM managers/project manager/managers, etc. (floor manager)}

The various managers create, oversee and instils these processes, continually auditing the production line to see how well it is performing and how well the processes are understood. This task is crucial for the production line to run smoothly. Above all, all employees must understand the importance of the processes and task responsibility for their workflow, so they can identify potential improvements and alert the managers to any issues.

\subsection{Architects/BIM modelers, etc. (floor staff)}

Anybody who interacts with the models/drawings is a part of the floor staff on the production line. Each person has a different, well-defined role and level of responsibility. As any task should be well documented and relatively easy to follow, anyone can pick up that task and perform it as necessary when they have completed their previous task.

Regular resourcing meetings should be scheduled to discuss who and what is available, and to make sure other floor staff are aware of this too. It is useful to review and discuss the weeks resourcing accuracy and effectiveness. When this is communicated effectively, tasks will be completed in a more purposeful manner, as the end goals of each task are more clearly outlined alongside the timeframe available.

\subsection{Document control (quality assurance)}

The document manager resides at the very end of our production line, performing the documented QA checks on each item that passes by and rejecting anything that is not up to scratch.

\subsection{Summary}

Ultimately, our findings show that the most effective improvements are those that are made on bottlenecks. Any other improvements are an illusion.

Having processes well documented stops the worst kind of bottlenecking; a person who does everything and nothing, a person who slowly becomes unreplaceable and who is always busy. 


\section{DOCUMENT MANAGEMENT}

Using the above best practice methodology, we then started reviewing the World Cup Final Stadium in context. This exposed some interesting facts about many of our current processes. They were not scale-able. As with many Architecture practices, many of the go between tasks fall between the cracks and were not documented or considered.

The size of the project was one that we had not previously encountered, and transformed normally simple, everyday tasks into ones that were unfeasible and onerous at scale. This was despite how well we considered the documentation and planning of our processes. A key example was the issue with the input and execution of PDFs, which have previously been completed manually.

This manual approach was not possible when converting 400 Lusail drawings into PDF, where each file could take 10 minutes to complete. Conversion was therefore an enormous task that would take an entire weekend and did not benefit from any sort of quality assurance. The potential for error was unacceptably high and the pressure on both person and time resource extremely inefficient.

The BIM team created a script to batch print drawings from Revit with the correct naming convention and paper size automatically [1]. This method significantly improved our quality processes by ensuring every drawing was correct and was compliant with the client's stringent BIM requirements. Furthermore, the ease of the process meant that any team member could use and run the script overnight with occasional checks via Remote desktop (RDP) if necessary. This simple change saved a significant amount of time and cost.

\subsection{Model standards and labelling}

Knowing there would be quite possibly hundreds of models between contractors and ourselves, not to mention the tens of thousands of drawings that would be created, it was imperative to agree on a standard. We were already working to PAS 1192 on most projects as were many of our contractors. Therefore, this seemed the obvious choice (since the time of writing this it has been made into an international standard: ISO 19650) [2].

To solve the issue of collaborating with numerous external consultants, we aimed for a method that would suit every user and every discipline in multiple countries and that would help us in managing a design that would change every single day. We opted for a service (BIM 360) that hosted our 3D models on the cloud. This service also allowed every other consultant to host and link their models together on the cloud.

We communicated problems in our federated model review software Navisworks. This meant we could jot down a clash or design issue and have an updated model from the contractor within the hour.

\section{CHALLENGE DESIGN: COMPUTATIONAL DESIGN VS. NORMAL DESIGN}

Performing manual tasks on a project of this size is impossible. What was needed was to remove the human element from the most onerous and resource-heavy activities, allowing the architect to concentrate on the tasks that will have the greatest quality impact on the project. This was achieved through computational design.

Computational design is the philosophy of abstracting a problem: breaking it down into its component parts and stepping away to provide perspective. By providing set constraints, we can allow computational refinery to find the optimum solution [3].

A client requires 80,000 people sat around a football pitch. Every person must have a minimum $\mathrm{C}$ value [4] of 60 and the volume of concrete used for the stands should be as low 
as possible. We can start by creating a mathematically driven parametric model for a stadium bowl that can bend, flex and resize.

Using computational design software with inbuilt optimisation algorithms (NSGA-II [5]) we can move adjustable values around, such as bowl curvature, offset from pitch corners and step height. This may then optimise the outputs, $\mathrm{C}$ values and volume of concrete for the bowl.

To make sure we have the optimal solution, the software will run many iterations. That is, the same algorithm will be run multiple times, slowly moving the values around until a set number of solutions are achieved. As an architect we can then choose our options by weighting our priorities.

For the project, we created an algorithm that produced the entire stadium bowl to the constraints of the brief and relevant regulations. We then tested the sightlines/seat kills. Further improvements can be made to this process with the use of Refinery, a Dynamo addin that takes these inputs and goals and runs the script multiple times to find the optimal solution.

Computational design has incredible functionality empowering designers to remove manual execution and manual calculations from the design process and allowing the designer to contribute their unique talents. The benefits are reducing material plus resources used, and significant time and cost saving.

\section{COMPETITION STAGE}

\subsection{Objective}

So far, we have explored the foundational principles of the processes used to deliver this project. This section will concentrate on the solution that secured our place in the design team: taking the original concept design and proving that is was deliverable within the client brief.

This was the solution that reduced the cost of the stadium by $40 \%$, allowing the project to continue with all original specifications intact.

\subsection{Challenge}

In the first instance, we analysed the information provided by the previous designers and studied the documentation provided by the client (BEP, EIR, etc. [6]). This package of information was cross-referenced with FIFA international standards [4].

What we discovered was that although the stadium was fully compliant with FIFA standards, the EIR had the more challenging constraints and therefore took precedence further challenging what could be achieved within the design.

From the EIR, we identified the design did not follow the client's documentation. This led us to make the following decision: revise the entire concept to fully comply to FIFA requirements and achieve the most efficient shape possible.

We were therefore confronted with the process of auditing an 80,000-seat stadium, creating a more efficient design and proving it was the winning option in a very short period of time. 


\subsection{Solution}

Simply, we understood the shape of the stadium would have the biggest impact on project cost. Achieving the most efficient bowl and therefore the most efficient overall footprint was the fundamental element that would be the difference between viability and failure.

The design of a bowl involves an enormous technical list of requirements such as:

- C-values [4];

- Net capacity [4];

- $\quad$ Seat kills [4];

- Obstruction sightlines [4];

- Pitch [4];

- Seating space [4];

- Seating categories [4] (general admission, VIP, media, disabled, etc.);

- $\quad$ Tread [4].

Again, we can turn to computational design for the solution.

Through an extremely complex visual programming script (Fig. 1) using languages such as Python, C\#, etc., where all the parameters from the EIR and FIFA document are considered, the $\mathrm{PC}$ ran a specific number of iterations selected by the designer. This resulted in a shortlist of the five most efficient options from a full long list of 100 .

All options were provided not just with geometry but with a huge quantity of data as maximum height, minimum height, number of seats, quality of seats, perimeter, etc. This information, when cross-referenced with FIFA standards, the EIR and the client documentation, was critical to make the right decision.

For example, the computational design was able to reduce the perimeter within a range of $1 \mathrm{~m}$ to $15 \mathrm{~m}$. This is turn reduced the façade and roof area. As designers, we ultimately chose $10 \mathrm{~m}$, being cognisant of future collaboration with other disciplines and being at the time unaware of their specific space requirements and constraints.

Time restrictions meant that we were unable to recreate the final envelope geometry by drafting in $2 \mathrm{D}$.

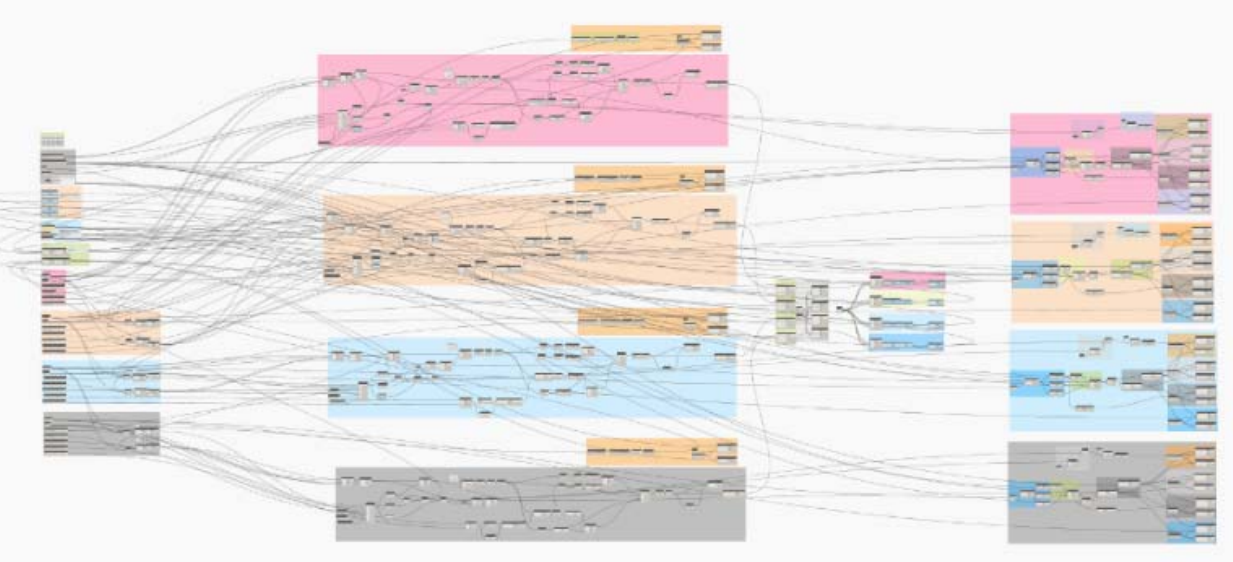

Figure 1: Graphical scripting. 


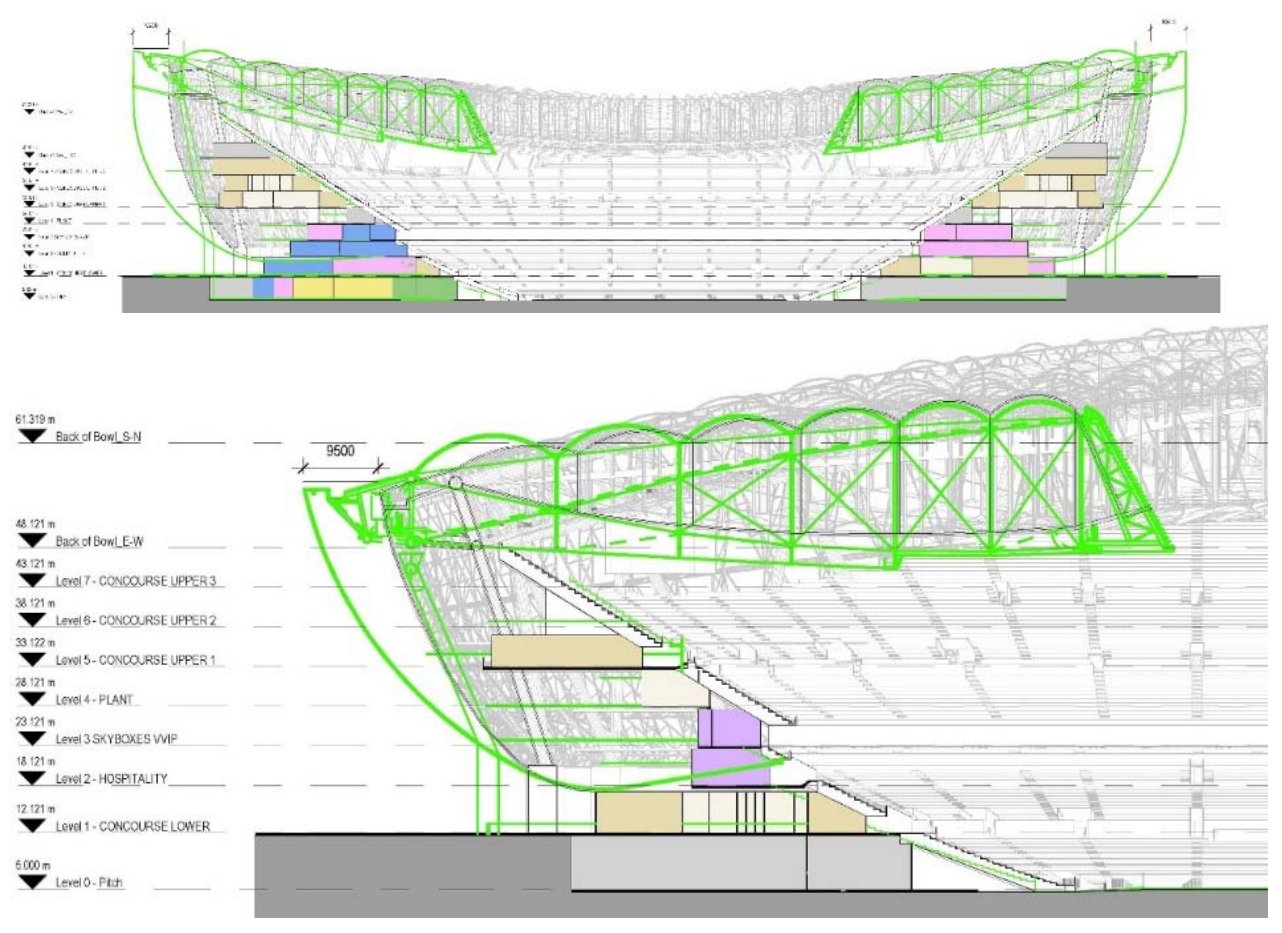

Figure 2: Geometry improvement.
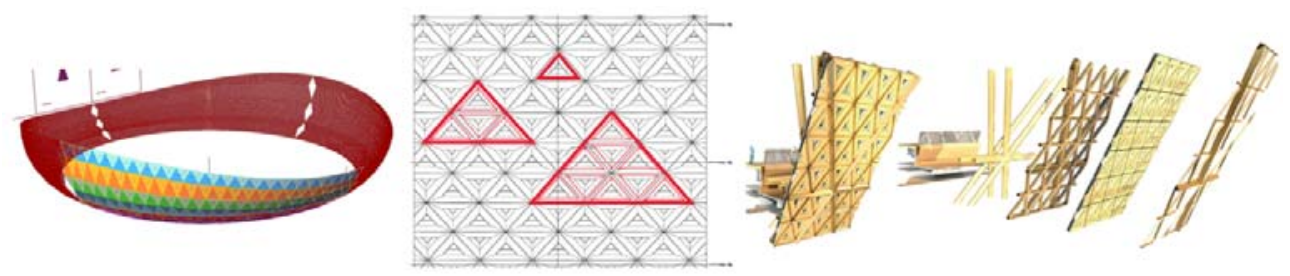

Figure 3: External envelope design.

Once we had chosen the most efficient geometry design, it was time to redevelop the external envelope; façade and roof (Fig. 2).

To recreate the resulting bowl geometry with $2 \mathrm{D}$ drafting is impossible within the time provided. Therefore, the computational design assisted us in that challenge.

Using the same strategy as the bowl, by a computational design process, we achieved the most efficient options (panels types, dimension and position them). It allowed us to reduce a previous design of $56,470 \mathrm{~m}^{2}$ of panels to the below picture $37,978 \mathrm{~m}^{2}$, which means a $35 \%$ reduction of external envelope materials.

The roof followed the same strategy as the façade but following an intersection of plane. 


\section{DESIGN STAGE}

Finally, the human element, presenting our discoveries and efficiencies to the client. Virtual reality in this case illustrated our solution effectively, allowing a full understanding of what we had achieved and the process we took to get to that stage.

\subsection{Objective}

From here, we were challenged to develop the concept design in four months, achieving an accurate net capacity as well as an accommodate schedule which complies with FIFA standards.

\subsection{Challenge}

To foresee in the design stage elements that should be considered in the construction stage such as metal work, advertising boards, and lighting.

\subsection{Solution}

At this stage, the most important facet of the stadium design is achieving the required net capacity. Further complicating this task is reducing the maximum number of sightline obstructions and achieving the combined requirements of both the EIR and FIFA standards regarding $\mathrm{C}$-values.

This required a full audit of the new bowl geometry as well as the functional spaces in the stadium. Combining trigonometric math with geometry, this output can be achieved as well as being able to be contained in the final model for handover.

The workflows we created included: heat map, seat kills and FIFA checklist [2].

\subsection{Heat map}

At this stage everything is about geometry and information that can be analysed. This workflow allows the client to know the exact quality of view per each spectator according to multiple constraints such as: seat type, thread, riser height, vertical height to the point of focus, etc.
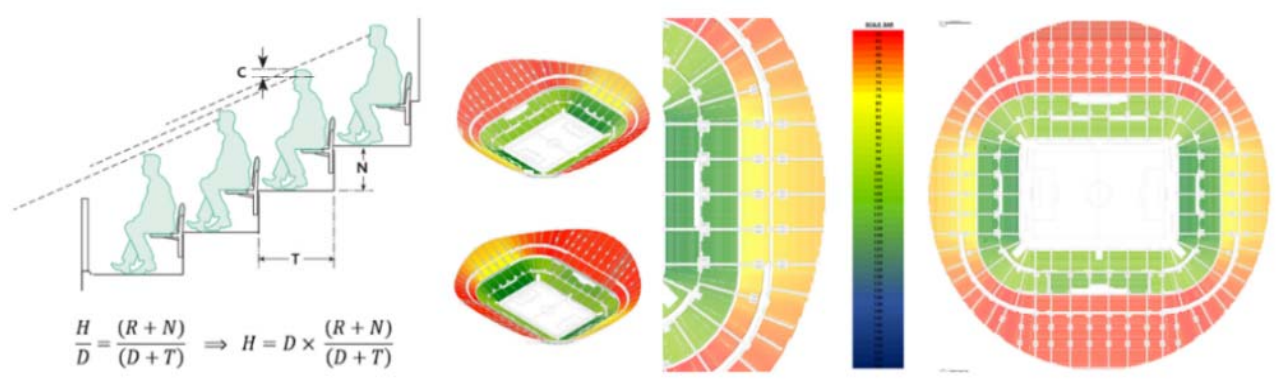

Figure 4: C-value, sightline quality representation. 


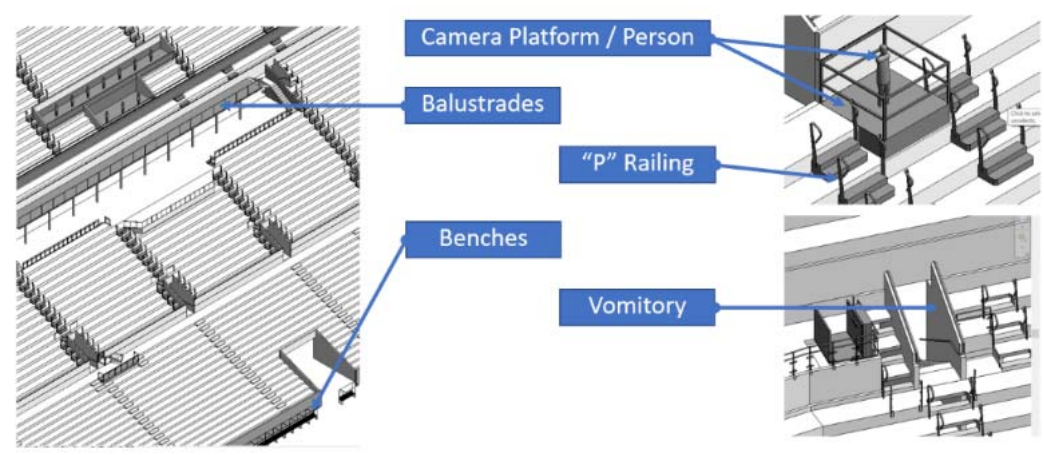

Figure 5: Obstruction types.

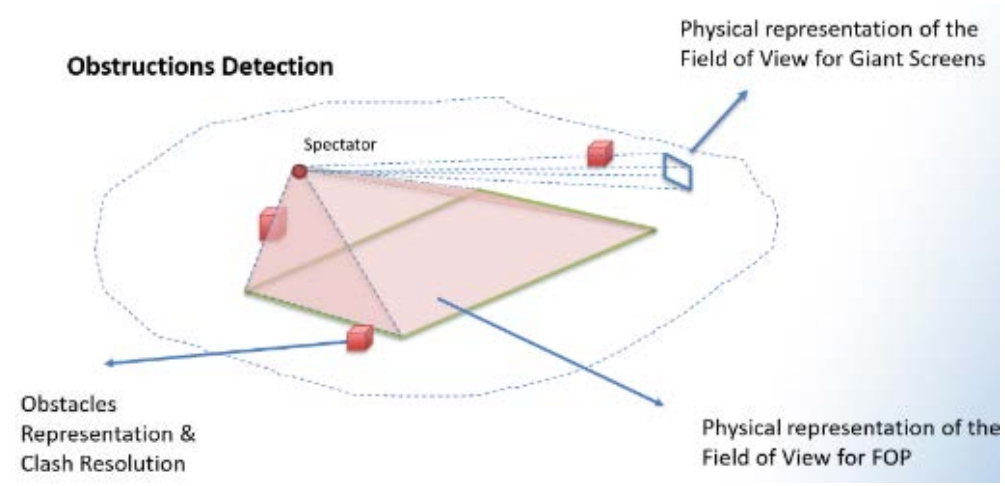

Figure 6: Modelling conditions.

\subsection{Seat kills}

Through a simple workflow supported by multiple softwares, we could foresee the exact quantity of spectators who will have an obstruction during events in the stadium. The process is as follows:

- Adding obstructions through computational design: Stadiums follow a very constrained geometry which took precedence at the concept stage. Through an algorithm in Dynamo we were able to insert obstacles such as advertising boards, benches, media screens, lighting, railing, vomitorium and gangways.

- Controlling the eye position of every single spectator: Each spectator has different constraints.

- Range of vision: By simulating each spectators' field of view.

- FIFA Constituent groups: Categorizing each group by their geometric constraints, i.e. a wheelchair and media seat have different eyelines.

- Clash detection: Identify any interference within the sightlines.

- Representation: To show the result visually as well as virtually, we recreated the experience of each spectator in the stadium. 


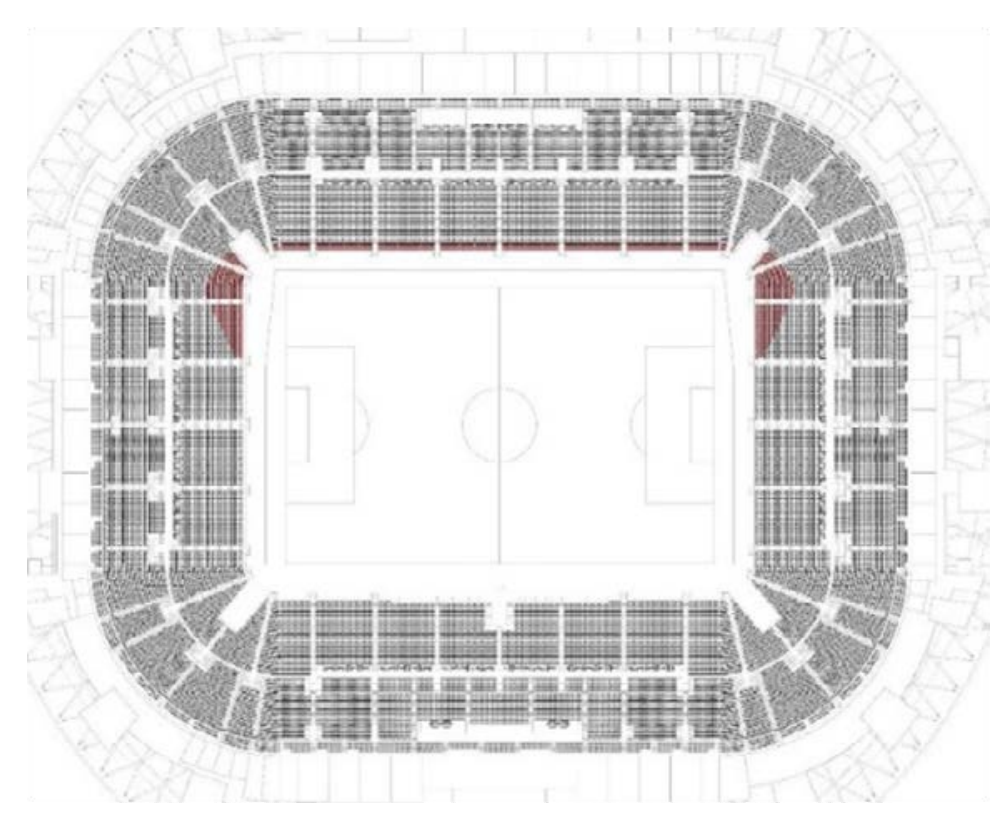

Figure 7: Obstructions detection.

Creating an index data base from EIR and FIFA allowed us to establish, maintain and audit the design each time the project is shared, therefore improving accuracy and control of the design on multiple platforms.

The database work is a symbiosis of two concepts: data and geometry. Manual errors happened on a daily basis, so this process allowed auditing the quality of the data as well as combining the information from 389 models into one, allowing any facility manager, client, contractor, mechanical engineer, structure engineer, to audit the information and get an easy understanding in a few pages of what the whole project offers.

The above is impossible to achieve in the way we all us worked in the past. It is a lesson that we learned and a process that was continually improved upon through many iterations. The benefits of the above methods were recognised by both the design team and client.

\section{CONSTRUCTION STAGE}

\subsection{Objective}

Deliver the project digitally, as-built fully coordinated to LOD 350 [6] in the least time possible.

\subsection{Challenge}

At this stage with the combination of highly demanding digital requirements, time constraints, model size, IT issues, time zones, length of supply chain, etc. The accurate production with high levels of coordination is extremely difficult and there is one concept that is constant in any project: Information needs to be in the right place at the sight time and communicated to the right people to achieve success. 


\subsection{Solution}

\subsubsection{Digital requirements}

Easily the project can get lost in bureaucracy, this is the reason why a programme that prioritises milestones is critical and most importantly it needs to be escalated with clients to show the benefits.

The symbiosing of modelling strategy with IT specification with design timescale has impact in the project and it needs to be considered beforehand.

\subsubsection{LOD 350}

In term of modelling it is a high detailing requirement especially when combined with the size of the project. The resulting solution was to split the project into 386 models, with all the resulting implications for drawings, data, and coordination.

\subsubsection{Time constraints/time zones/different locations}

Due to the size of the project and the timescale, sharing the project between offices was essential for efficient working. While an office is sleeping another office can carry on working during the day and can work in the same model strategy at the same time. Therefore, working via a cloud platform, we could achieve BIM Level 3 [6].

\subsubsection{Coordination}

One of the most difficult barriers we were facing was the slow connectivity speed in Qatar, an issue when trying to exchange models and thousands of drawings with site. The solution we used for this situation, was Dropbox. The way it incrementally syncs through the desktop allowed site to receive the files. However, this created a breach in QA and other processes so by creating an internal infrastructure of process document management control when all our office was connected via a mix of digital platform was essential.

Another key subject was the coordination of a live design day by day. How to manage clashes and coordinate the experience design. To understand what is happening in a project of this size considering all disciplines is extremely difficult, and there is a high risk that disciplines are not well coordinated. A weekly review/walkthrough of the model was essential with all parties present.

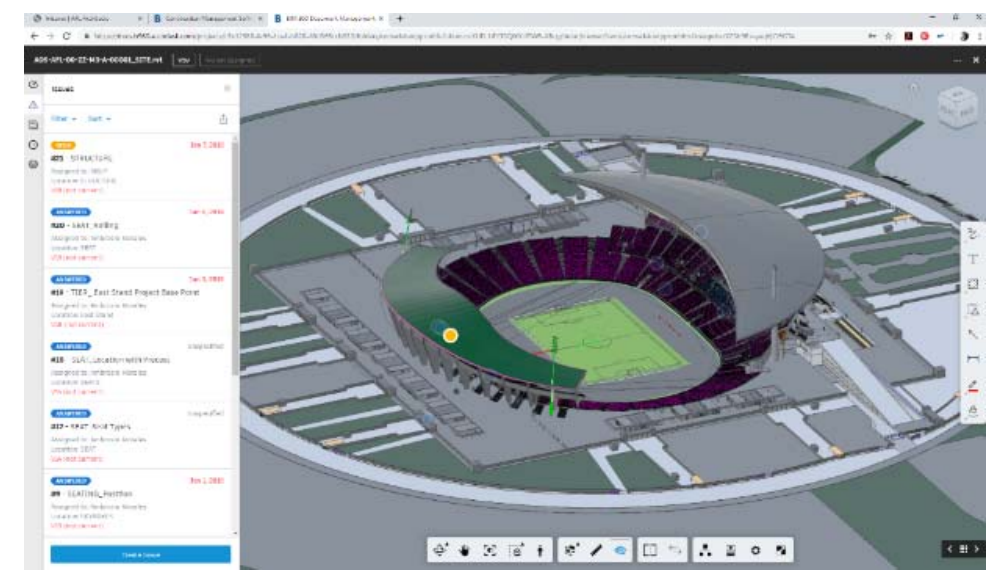

Figure 8: Working collaborative in the cloud. 


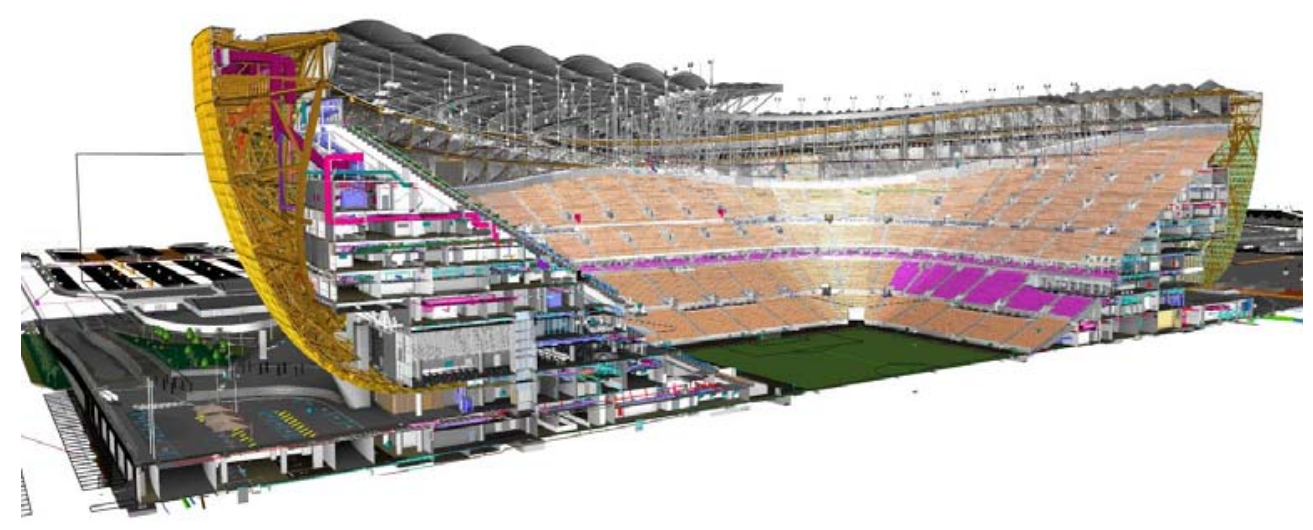

Figure 9: Coordination with all disciplines.

To smooth this process, all the coordination issues were stored in the cloud where everyone could have access through Autodesk Navisworks, also a range of metadata attributed to each issue:

- Priority: The designer and contractor should focus on each one according to the programme. To resolve all of them at the same time is not efficient.

- Location: Issues are geolocated, but data is essential to be added for order and searching purposes. It is important for construction stage as it allows the designer and contractors to focus in the construction zones/phases through the timeline.

- Package: Designer and builder work with the package of work to build the project, so it is key to manage the issue through the programme.

- Discipline: Often issues have involved and not specific disciplines, by having information on a big range is beneficial also happens that designers are relocated in different packages/phases/zone, it allows everyone to be on the same page.

- Author: To track an issue is essential to know who highlighted the issue.

- Description: Through a picture and description of what the issue is make simple to understand in any situation.

- Contact/s to answer: It is fundamental to have a detailed organigram of who is responsible of allowing to assign the right person the issue as it speeds the process otherwise issue can get lost in time and desperate the team. The goal in all this process is to have the right information in the right place at the right time.

- Comment: It allows to reply, answer or have a live conversation about the issue from anywhere in the world.

\section{CONCLUSION}

It is not every day that an architecture practice is given a project ten times bigger and more complex than they have ever done before. But in theory, it should not be ten times more difficult. The processes and workflows used in a practice should be able to scale and adapt. We spent a long time improving workflows throughout this project and our key development was that any improvements should be made on the bottlenecks of the workflow. Any other 
improvements are an illusion. That is, identifying precisely where in the process the most slowdown is being experienced and concentrating resources on a solution.

It is critical to any practice to incentivize improvement and to inspire people to work out better ways of doing things. "It is the way we have always done it" is not a sustainable workflow methodology and will constantly close doors and possibilities. With the right time and effort concentrated on bottleneck tasks, a practice will benefit in the long term and remain sustainable into the future.

\section{ACKNOWLEDGEMENT}

We thank Claire Gilchrist for acting as copywriter and for her comments that greatly improved the article.

\section{REFERENCES}

[1] Sobon, K.K., Printing PDFs w/ dynamo, 2015. http://archi-lab.net/printing-pdfs-w-dynamo/. Accessed on: 20 Jun. 2019.

[2] BSI Group, What is ISO 19650? 2019. www.bsigroup.com/en-GB/Building-Information-Modelling-BIM/bim-design-andconstruction/iso-19650/. Accessed on: 10 May 2019.

[3] Rutten, D., Evolutionary principles applied to problem solving, 2010. www.grasshopper3d.com/profiles/blogs/evolutionary-principles. Accessed on: 26 May 2019.

[4] Football Licensing Authority, Guide to safety at sports grounds, 5th ed., 2008. www.raithrovers.net/files/GuidetoSafetyatSportsGrounds.pdf. Accessed on: 26 Jun. 2019.

[5] Kalyanmoy, D., Pratap, A., Agarwal, S. \& Meyarivan, T., A fast and elitist multiobjective genetic algorithm: NSGA-II. IEEE Transactions on Evolutionary Computation, 6(1), pp. 182-197, 2002.

[6] British Standards, British Standards and Publicly Available Specifications (PAS) from BSI, 2007-2018. https://bim-level2.org/en/standards/. Accessed on: 24 Jun. 2019. 